\title{
Circulatory support with attenuated pulse pressure alters human aortic wall morphology
}

\author{
Stephen Westaby, FRCS, MS, PhD, FETCS, FESC, FACC, FICA, ${ }^{a}$ Gabriele B. Bertoni, MD, ${ }^{\text {a }}$ Colin Clelland, FRCPath, \\ Tomohiro Nishinaka, MD, PhD, a and O. H. Frazier, MD, ${ }^{b}$ Oxford, United Kingdom, and Houston, Tex
}

$\mathrm{I}$ n biology "form follows function." Hypertension causes the musculoelastic aortic wall to thicken. ${ }^{1}$ The only situation characterized by chronic hypotension with diminished pulse pressure is in patients having long-term support with a left ventricular assist device (LVAD). In a sheep model of chronic nonpulsatile blood flow, the medial layer thinned with apoptosis of smooth muscle cells (SMCs) and fragmentation of elastic fibers. ${ }^{2}$ We sought to determine the clinical implications of this finding for destination therapy patients with a rotary blood pump.

\section{Clinical Summary}

In 7 patients with end-stage heart failure, computed tomographic scans were taken before implantation of a Jarvik 2000 LVAD (Jarvik Heart, Inc, New York, NY) and then annually during survival. Preoperative and latest postoperative aortic diameters were compared. After 5 deaths at between 7 days and 34 months of support, we obtained multiple full-thickness sections of the descending thoracic aorta. At least two nonadjacent sections were processed for each patient. The samples were fixed and stained with hematoxylin and eosin (H\&E) and elastic van Gieson (EVG) stains. Control samples were taken from nonvasculopathic cadavers of similar age, sex, and body mass. These were obtained by our homograft valve bank with consent in accordance with the Human Tissue Act (United Kingdom).

Thickness of the aortic medial layer was measured from an average of 35 nonadjacent fields $(\mathrm{EVG}$, magnification $\times 40)$. SMCs were counted from an average of 20 non-adjacent fields $(H \& E$, magnification $\times 200)$ per patient. Data were analyzed with StatsDirects (version 2.5.7; StatsDirect Ltd, Sale, Cheshire, United Kingdom) and presented as median $\pm \mathrm{SD}$. Analysis of variance was calculated using 2-way analysis of variance with Bonferroni comparison.

None of the computed tomographic scans showed an increase in aortic diameter out to 6 years (Figure 1). The 7-day LVAD specimens were indistinguishable from controls, but distinct morphologic changes were evident in the 3-month samples. Medial

From Oxford Heart Center, ${ }^{a}$ John Radcliffe Hospital, Department of Cardiothoracic Surgery, Oxford, United Kingdom; and The Texas Centre, Houston, Tex.

Received for publication Oct 5, 2006; accepted for publication Oct 9, 2006.

Corresponding Author: Prof. Stephen Westaby, FRCS, MS, PhD, FETCS, FESC, FACC, FICA, Oxford Heart Center, John Radcliffe Hospital, Department of Cardiothoracic Surgery, Headley Way, Headington, Oxford, Oxfordshire Ox3 9DU, United Kingdom (swestaby@AHF.org.uk).

J Thorac Cardiovasc Surg 2007;133:575-6

$0022-5223 / \$ 32.00$

Copyright $\odot 2007$ by The American Association for Thoracic Surgery doi:10.1016/j.jtcvs.2006.10.014 thickness diminished during prolonged support $(1.411 \pm 0.140$ $\mathrm{mm}$ in controls vs $1.006 \pm 0.170 \mathrm{~mm}$ in LVAD patients; $P<$ $.0001)$. Medial SMCs decreased in number (147 \pm 31 per field in controls vs $63 \pm 12$ per field in LVAD patients; $P<.0001$ ) (Figure 2). From the EVG-stained sections there was a concomitant decrease in the medial layer elastin content. The process of atrophy appeared to progress with time.

\section{Discussion}

These novel findings were predictable but have not previously been confirmed in humans.

One study limitation is that the autopsy samples could not be compared with the same patients' aorta obtained during LVAD implantation. However, we were initially unaware of the sheep findings from our Terumo ventricular assist device program. ${ }^{2}$ It was this that triggered the human study. Matched controls were the alternative. Irrespective of this, the human changes closely parallel the laboratory findings. ${ }^{3}$

Arteries remodel in response to pressure and flow to provide a constant level of shear stress. Remodeling follows a complex interaction between signaling pathways and altered gene expression for nitric oxide synthase, platelet-derived growth factor, and transforming growth factor $\beta_{1} .{ }^{4}$ Because the Jarvik 2000 LVAD is afterload sensitive, mean systemic arterial blood pressure (MAP)

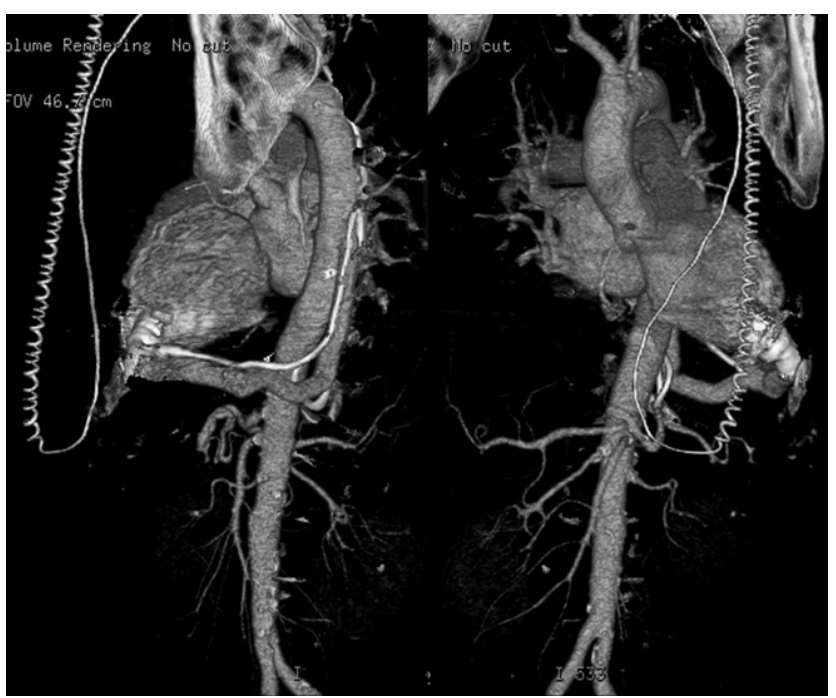

Figure 1. Computed tomographic scan from a surviving patient 6 years after implantation of a Jarvik 2000 LVAD. There are no changes in size at any level compared with the preoperative scan. Preoperative scans are performed routinely to rule out disease at the site of vascular graft anastomosis. 


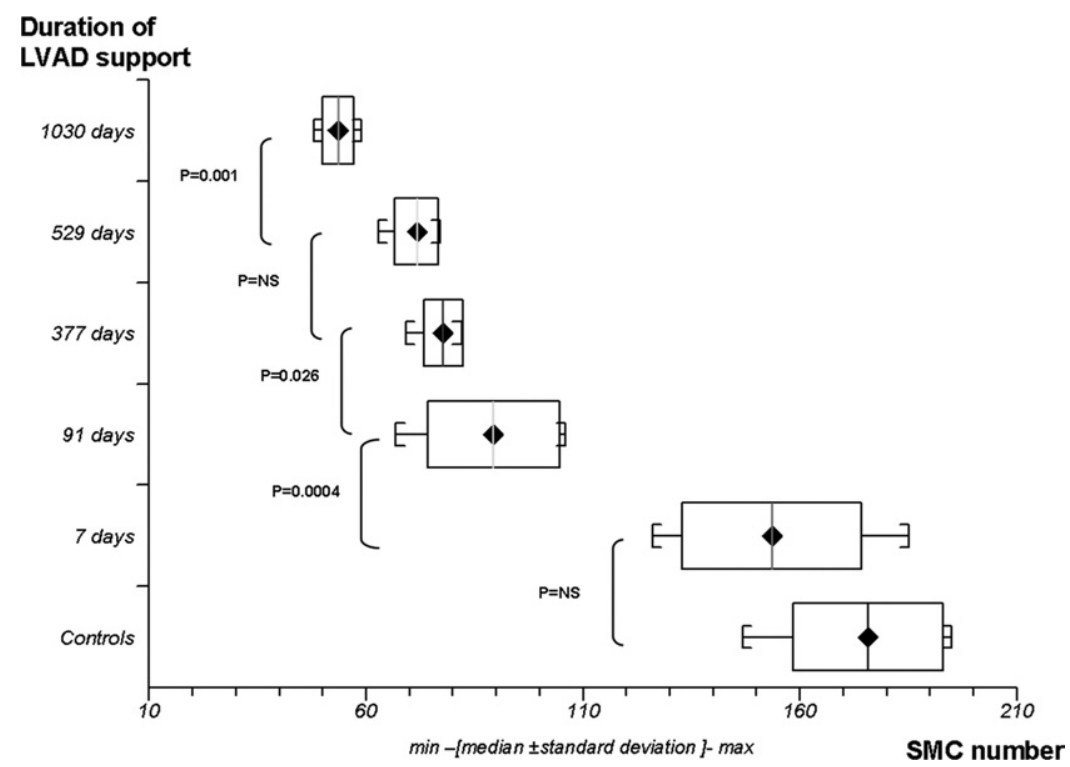

Figure 2. SMC count from the aortic medial layer at different durations of support compared with control samples. There is a statistically significant decrease in medial SMCs, which appears to progress with time.

was pharmacologically reduced to 70 to $90 \mathrm{~mm} \mathrm{Hg}$. Attenuated pulse pressure between 10 and $20 \mathrm{~mm} \mathrm{Hg}$ is usually detectable through left ventricular contraction. In these circumstances, shear stress is reduced through both nonpulsatile pump flow and MAP reduction.

In hypertension, aortic hypertrophy results from stimulation of angiotensin I receptor, which promotes SMC growth and inhibits apoptosis. Angiotensin I receptor blockade (eg, losar$\tan$ ) induces a transient burst of SMC apoptosis and regression of medial hypertrophy. ${ }^{5}$ Angiotensin II receptors mediate vascular mass regression by SMC apoptosis in vivo. In animal models we and others have shown that SMC apoptosis occurs in response to reduced arterial wall tension, suggesting that blood pressure-dependent and angiotensin-independent apoptotic pathways are possible. ${ }^{3}$ As well as medial thinning and diminished SMC number in goats, Nishimura and associates ${ }^{3}$ showed an elevation in the number of inactive (atrophic) SMCs with increased density. Atrophy of SMCs has also been demonstrated in other models of hypotension, decreased aortic flow, and hypoxia. Perhaps because of MAP control, the LVAD patient's aorta does not dilate during these morphologic changes.

Evidence shows rotary blood pumps to be user friendly, safe, and effective for prolonged periods. The aortic wall changes are not likely to achieve clinical significance. On the contrary, the potential therapeutic benefits of pulse pressure modulation in vasculopathic patients are compelling. Virtual elimination of pulse pressure may prevent progression or even reverse arterial disease or may prevent aneurysm rupture in poor surgical candidates. This approach may prove feasible with the development of even smaller devices that can be implanted without major surgery.

\section{References}

1. Owens GK. Influence of blood pressure on development of aortic medial smooth muscle hypertrophy in spontaneously hypertensive rats. Hypertension. 1987;9:178-87.

2. Saito S, Westaby S, Piggot D, Dudnikov S, Robson D, Catarino PA, et al. End-organ function during chronic nonpulsatile circulation. Ann Thorac Surg. 2002;74:1080-5.

3. Nishimura T, Tatsumi E, Takaichi S, Taenaka Y, Wakisaka Y, Nakatani $\mathrm{T}$, et al. Prolonged nonpulsatile left heart bypass with reduced systemic pulse pressure causes morphological changes in the aortic wall. Artif Organs. 1998;22:405-10.

4. Pollman MJ, Yamada T, Horiuchi M, Gibbons GH. Vasoactive substances regulate vascular smooth muscle cell apoptosis countervailing influences of nitric oxide and angiotensin II. Circ Res. 1996;79:748-56.

5. Marchand EL, Der Sarkissian S, Hamet P, deBlois D. Caspasedependent cell death mediates the early phase of aortic hypertrophy regression in losartan-treated spontaneously hypertensive rats. Circ Res. 2003;92:777-84 